\title{
Evaluation of microshock risk during a surgical procedure
}

\author{
G. L. Amicucci ${ }^{2}$, E. Zennaro ${ }^{1}$, F. Fiamingo ${ }^{2}$ \& C. Mazzetti ${ }^{1}$ \\ ${ }^{1}$ Department of Astronautics, Electrical and Energetics \\ Engineering (DIAEE) Electrical Engineering Section, \\ Sapienza Università di Roma, Italy \\ ${ }^{2}$ Department of Safety Technology (DTS), \\ Research, Certification and Verification Sector, \\ Istituto Nazionale per l'Assicurazione contro gli Infortuni \\ sul Lavoro (INAIL), Italy
}

\begin{abstract}
During a surgical procedure, risk assessment is important in order to prevent undesired patient's ventricular fibrillation. Even weak currents flowing through the heart of a patient are potentially able to cause such a dangerous event.

The hazard can be reduced through the adoption of suitable protection measures, chosen by means of an in-depth investigation of the microshock probability.

Such a probability is obtained by the product of the probability of ventricular fibrillation when certain fault conditions occur and the probability of occurrence of the considered fault conditions. The fault conditions here considered are able to determinate certain values of the leakage current that can flow through the patient's body.

A method for the microshock probability assessment is presented based on the leakage current estimation that is obtained by simulation of an electrical circuit model of a typical surgical layout.

The layout in question is composed of an operating table, a patient monitor and a defibrillator; all supplied by a medical IT system. Leakage currents flowing from this medical equipment to the chest of the patient, are measured in normal and in fault conditions. The permitted leakage current values are obtained by the international standard IEC 60601-1, and an estimate of the probability of
\end{abstract}


ventricular fibrillation at different values of the leakage current is obtained by the Annex A (rationale) of the same standard.

Keywords: microshock risk, defibrillator, operating table, patient monitor.

\section{Introduction}

Operating theatres are workplaces where the protection from electrical injuries is critical. In fact several Medical Electrical Equipment (MEE) are used by medical workers and are in contact with the patient in order to perform their function.

During open heart surgery and when catheters are in use, it is necessary to minimize leakage currents that may flow through the patient. Current as low as $10 \mu \mathrm{A}$ through the heart may be fatal causing ventricular fibrillation (microshock).

To prevent risks, power systems supply must operate with a high degree of reliability and quality. A safe use of MEE can be accomplished by selecting proper circuit mitigating interventions $[1,2]$.

In literature some microshock events are reported (see for instance [3-5]). International standards give information on the ventricular fibrillation probability.

Such a probability depends on the current that flows through the heart.

During a surgical procedure or when a MEE is applied to a patient for diagnosis or therapy, a fault condition could eventually determinate values of the leakage current flowing through the patient by the MEE that are potentially dangerous. Hence, the microshock probability can be evaluated by the product of the probability of ventricular fibrillation and the probability of occurrence of the considered fault conditions, as discussed in [6].

The simulations presented in the paper have been used to quantify the microshock probability and, consequently, to identify which interventions can reduce the risk under a tolerable level.

In a previous paper [7], a method for the modeling of a safety oriented electrical circuit of a defibrillator and the results of leakage current simulations were shown. The usefulness of those simulations is explained in the present paper which extends the simulation to the electrical circuit model of a surgical layout composed by an operating table, a patient monitor and a defibrillator, all supplied by a medical IT system. The circuit model is deeply explained in section 2. In section 3 the limit values of leakage currents evaluation in fault conditions are considered. In section 4 the microshock probability is evaluated and in section 5 the results of such evaluation are presented and discussed. In section 6, some conclusion and further development of the work are formulated.

\section{Surgical layout circuit model}

\subsection{Electrical circuit model of medical equipment}

In the paper [7] the electrical circuit model of a MEE has been obtained by using electrical safety tests on a real MEE and information on electrical insulations. 
The tests have been accomplished according to the procedure described in the applicable international standards $[8,9]$.

Medical equipment modelled in this work are a patient monitor, an operating table and a defibrillator. The first two MEEs are always involved in surgical procedures, the third equipment can be put into operation during emergencies, for example, when ventricular fibrillation occurs. So, we draw a circuit model of a typical surgical layout.

Electrical circuit models of three MEE are obtained by results of a campaign of measures accomplished by the Clinical Engineering Unit (CEU) of the hospital Campus Bio-Medico in Rome in accordance with the measurement setup described in the international standard EN 62353 [9]. In particular, equipment leakage currents (ELCs) and applied part leakage currents (APLCs) are obtained by the direct test method described in the standard. The circuits are simulated with Multisim a software by National Instruments.

The patient monitor modelled is a class I MEE which can be supplied with $230 \mathrm{~V} \mathrm{AC}$. It is composed by six floating type applied parts (CF-type and BFtype) which can be divided in three groups considering the different functions performed. They are the ECG monitoring by CF-type electrodes, the oxygen saturation monitoring by the BF-type pulse oximetry and the blood pressure monitoring by the BF-type sphygmomanometer.

The operating table is a class I equipment with body type (B) applied part, i.e. the table top where the patient lies during the surgical procedure.

The defibrillator is a class I equipment with three CF-type applied parts which perform the ECG monitoring and two BF-type applied parts which are the defibrillator electrodes, its model was already presented in the paper [10].

The values of the electrical parameters and the simulated leakage currents of each MEE alone, i.e. considered by itself, are not reported since they don't differ significantly from those reported in [7]. The model of the complete layout, instead, is presented here for the first time.

\subsection{Electrical circuit model of a medical IT supply system}

There are suitable measures preventing faults which can be applied to electrical system and equipment in an operating theatre in order to reduce the microshock risk, as it is shown in [2]. The two main mitigating interventions are the medical IT system (IT-M), which supplies MEE by isolating the secondary windings of the transformer from the earth constituted by the equipotential bonding bar, and the equipotential bonding between equipment connected to the patient that avoids the voltage drop and the eventual flow of dangerous leakage currents through the patient's body.

Moreover, the international standard IEC 60364-7-710 [11] fixes equal to $500 \mu \mathrm{A}$ the maximum admissible value of the earth leakage current of the unloaded isolation transformer. And the same standard fixes to $200 \mathrm{~m} \Omega$ the maximum admissible value of the conductor and connection resistances between the earth terminal of the mains plug and the equipotential bonding bus bar. Finally, the standard IEC 62353 permits that the maximum value of resistance between the earth terminal of the mains plug and the protectively earthed 
accessible conductive parts of the MEE be equal to $300 \mathrm{~m} \Omega$. Hence, in the circuit model of the surgical layout used in the simulations the PE conductors comply with such admissible values and the isolation to earth of the secondary winding is modeled with two stray capacitances of each phase conductor to earth [12].

\subsection{Electrical circuit model of patient}

According to [13], the patient is simulated by a network of five resistances representing left arm, R25, trunk, R51, left leg, R50, right arm, R1 and right leg, $\mathrm{R} 26$. The sum of three resistances belonging to one side of the human body is equal to the conventional value of $1 \mathrm{k} \Omega$. It is the same for the other side of the human body. Indeed a percentage of $47.2 \%$ of the total body resistance is assigned to one arm, which is mainly composed of bones, a $1.3 \%$ is assigned to trunk, assumed to be a cylinder full of saline water highly conductive with a discrete amount of bones, and a percentage of $51.5 \%$ is assigned to each leg, which is larger than the arm but with similar organic content.

Figure 1 shows the three MEE connected with the patient and supplied by a medical-IT system.

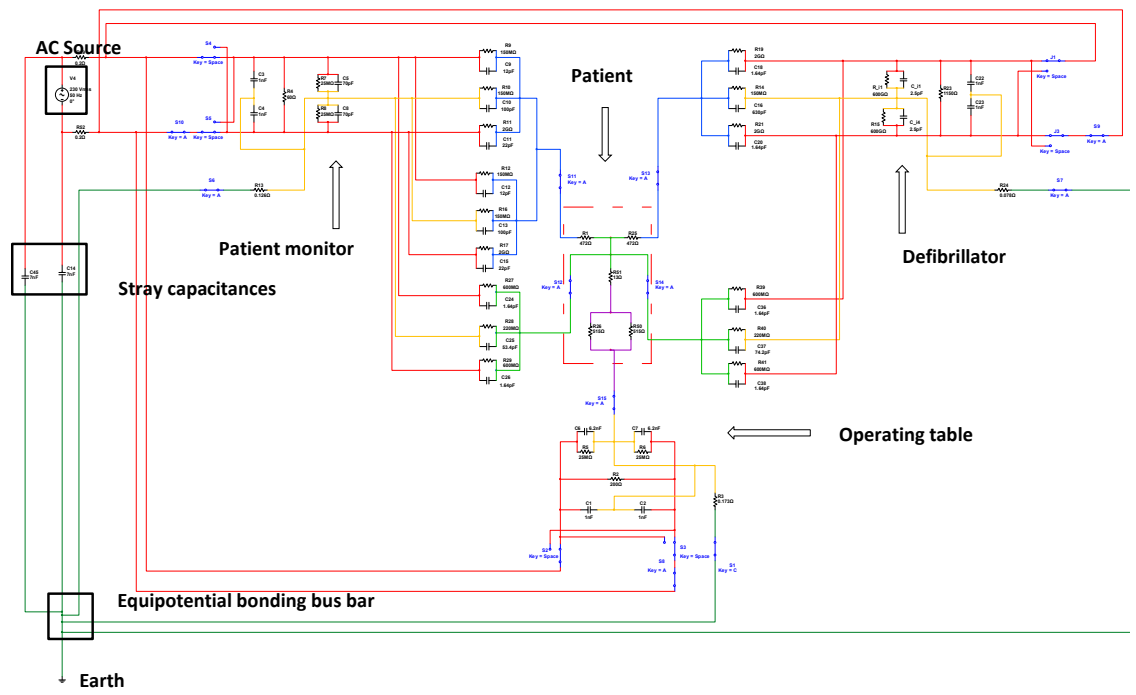

Figure 1: Electrical circuit model of three MEE in contact with the patient in an operating theatre. 


\section{Leakage currents evaluation in fault conditions}

\subsection{New limit values}

Limit values of leakage current reported by international standards are based on a series of studies.

In the present paper it has been taken as a reference the relationship between the values of the leakage current with the probability of ventricular fibrillation that has been presented in [14]. Values of current flowing through the heart of the patient leading to a probability of ventricular fibrillation less or equal than $1 \%$ are not considered as eventually dangerous. However, since similar threshold limits are chosen on a probabilistic base to be effective for a large fraction of the population, some authors consider as possibly dangerous also lower values [15]. In fact, currents lower than $50 \mu \mathrm{A}$ could be dangerous for patients, with some heart disease, during cardiac surgical procedures. In a study performed on 40 patients affected by several cardiac pathologies [16], an alternate current was given through a pacing catheter placed near the right ventricular apex. New limit values were recorded as potentially dangerous for patients. These are $20 \mu \mathrm{A}$, $32 \mu \mathrm{A}$ and $49 \mu \mathrm{A}$. These are the threshold values considered in this paper to underline the most dangerous fault conditions that could occur during a surgical procedure in an operating theatre.

\section{Microshock probability evaluation}

\subsection{Probability of ventricular fibrillation}

According to the reference [8] some ventricular fibrillation probability values are identified and reported in Table 1.

Table 1: Relationship between leakage current values and probability of ventricular fibrillation in fraction.

\begin{tabular}{ll}
\hline Current $[\boldsymbol{\mu A}]$ & Damage probability \\
\hline 20 & 0.003 \\
\hline 32 & 0.005 \\
\hline 40 & 0.007 \\
\hline 50 & 0.01 \\
\hline 60 & 0.015 \\
\hline 100 & 0.065 \\
\hline 150 & 0.25 \\
\hline 200 & 0.5 \\
\hline 250 & 0.78 \\
\hline 300 & 0.94 \\
\hline 1000 & 1 \\
\hline
\end{tabular}




\subsection{Probability of occurrence of undesired events}

According to the standard [8] some undesired events are identified and the occurrence probability values are reported in Table 2 . The probability of these events is evaluated in terms of number of surgical procedures. So a probability of 0,01 corresponds to 1 occurrence every 100 surgical procedures.

Table 2: Occurrence probabilities, in fraction, of determined undesired events.

\begin{tabular}{lc}
\hline Undesired condition & Occurrence probability \\
\hline Degradation of applied part insulation & 0.01 \\
\hline Double fault to one equipment & 0.01 \\
\hline Single fault to two equipment together & 0.01 \\
\hline Single fault to one equipment & 0.1 \\
\hline
\end{tabular}

The probability of occurrence of a single fault condition, as the fault of the PE conductor in a class I MEE, is considered equal to $10 \%$, while the probability of occurrence of a double fault condition is evaluated by squaring such value (i.e. by considering the failure of two insulations, or two means of protection, as independent events).

For example, taking into account a floating type applied part, its insulation should be considered as composed by two insulations whose failures are independent; the fault probability of both could be calculated by squaring 0.1 as reported in Table 2 .

\section{Results}

\subsection{Results of leakage current evaluation}

In this section the results of simulations of the circuit model shown in Fig. 1 are presented. The values of leakage current monitored at the chest of the patient are simulated in these conditions:

a) Normal condition, when three MEE operates without faults;

b) Single fault condition, when a mains part or the PE conductor linked with one MEE is disconnected;

c) Double fault condition, when the events in b) occurred together to the same MEE, or occurred singly to two of three MEE in a determined period of time;

d) Degradation of the insulations of one MEE;

e) Normal polarity of all equipment, inverse polarity of all equipment, and inverse polarity of each apparatus in turn.

Values of potentially dangerous leakage currents flowing through the resistance of the trunk, R51, are shown in Table 3 for condition c) and in Table 4 
for condition d) in the case of patient monitor. There are no relevant differences if one would consider the other two pieces of medical equipment.

Table 3: Leakage currents simulated in different fault conditions.

\begin{tabular}{lc}
\hline Fault condition & $\begin{array}{c}\text { Patient's chest leakage } \\
\text { current }\end{array}$ \\
\hline $\begin{array}{l}\text { Disconnection of a mains part } \\
\text { and disconnection of PE of the }\end{array}$ & $58,8 \mu \mathrm{A}$ \\
operating table & \\
\hline $\begin{array}{l}\text { Disconnection of a mains part } \\
\text { and disconnection of PE of the } \\
\text { defibrillator }\end{array}$ & $29,7 \mu \mathrm{A}$ \\
\hline
\end{tabular}

Table 4: Leakage currents simulated during electrical insulations ageing of patient monitor.

\begin{tabular}{|c|c|c|}
\hline \multicolumn{3}{|c|}{ BF-type applied part - MP1 insulation } \\
\hline Capacitance $[\mathrm{pF}]$ & Impedance[M $\Omega]$ & Current $[\mu \mathrm{A}]$ \\
\hline 12 & 130 & 1.57 \\
\hline 600 & 5.3 & 20 \\
\hline 900 & 3.5 & 30.2 \\
\hline 1500 & 2.1 & 50.1 \\
\hline Resistance [M $\Omega]$ & Impedance[M $\Omega]$ & Current $[\mu \mathrm{A}]$ \\
\hline 150 & 130 & 1.57 \\
\hline 10 & 10 & 12 \\
\hline 0.5 & 0.5 & 223 \\
\hline $0.051 *$ & 0.051 & 1020 \\
\hline \multicolumn{3}{|c|}{ BF-type applied part - MP2 insulation } \\
\hline Capacitance [pF] & Impedance[M $\Omega]$ & Current $[\mu \mathrm{A}]$ \\
\hline 22 & 144 & 1.57 \\
\hline 600 & 104 & 21 \\
\hline 920 & 0.5 & 31.9 \\
\hline 1450 & 0.051 & 49.5 \\
\hline Resistance [M $\Omega$ ] & Impedance[M $[$ ] & Current $[\mu \mathrm{A}]$ \\
\hline 2000 & 144 & 1.57 \\
\hline 150 & 5.3 & 1 \\
\hline 0.5 & 3.4 & 221 \\
\hline $0.051 *$ & 2.1 & 1020 \\
\hline
\end{tabular}


Table 4: $\quad$ Continued.

\begin{tabular}{|c|c|c|}
\hline \multicolumn{3}{|c|}{ CF-type applied part - MP1 insulation } \\
\hline Capacitance $[\mathrm{pF}]$ & Impedance[M $[\mathbf{]}]$ & Current $[\boldsymbol{\mu A}]$ \\
\hline 1.64 & 573 & 1.57 \\
\hline 600 & 5.3 & 20.3 \\
\hline 900 & 3.5 & 30.5 \\
\hline 1500 & 2.1 & 50.5 \\
\hline Resistance [M $\Omega]$ & Impedance[M $[\mathbf{]}]$ & Current $[\boldsymbol{\mu A}]$ \\
\hline 600 & 573 & 1.57 \\
\hline 150 & 149 & 2.10 \\
\hline 0.5 & 0.5 & 223 \\
\hline $0.051 *$ & 0.051 & 1030 \\
\hline
\end{tabular}

CF-type applied part - MP2 insulation

\begin{tabular}{|c|c|c|}
\hline Capacitance $[\mathrm{pF}]$ & 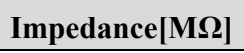 & Current $[\boldsymbol{\mu A}]$ \\
\hline 1.64 & 573 & 1.57 \\
\hline 600 & 5.3 & 21.7 \\
\hline 900 & 3.4 & 31.9 \\
\hline 1450 & 2.2 & 50.2 \\
\hline Resistance [M $\Omega$ ] & Impedance[M $\Omega]$ & Current $[\mu \mathrm{A}]$ \\
\hline 600 & 573 & 1.57 \\
\hline 150 & 149 & 1.10 \\
\hline 0.5 & 0.5 & 221 \\
\hline $0.051 *$ & 0.051 & 1020 \\
\hline $\begin{array}{l}* \text { Value just greater } \\
\text { by the insulatio }\end{array}$ & $\begin{array}{l}\text { shold value equal t } \\
\text { device [11]. }\end{array}$ & $50 \mathrm{k} \Omega$ signalle \\
\hline
\end{tabular}

\subsection{Results of microshock probability evaluation}

As anticipated in section 1 the microshock probability is evaluated by the product of the probability of ventricular fibrillation and the probability of occurrence of fault conditions.

In Table 5 the results of microshock probability are reported.

The tolerable limit for the microshock probability is assumed $10^{-3}$, as reported by the standard [8]. The degradation of the insulation between an applied part and a mains part, whose impedance goes down to a value of few kiloohms, lead to the greatest and not tolerable value of the microshock probability. The minimum value of the insulation resistance, leading to patient leakage current 
Table 5: Microshock probability values, according to the identified fault conditions.

\begin{tabular}{lll}
\hline & Fault condition & Probability of microshock \\
\hline 1. & Degradation of AP insulation to $0.05 \mathrm{M} \Omega$ & $10^{-2}$ \\
\hline 2. & Degradation of AP insulation to $0.5 \mathrm{M} \Omega$ & $5^{*} 10^{-3}$ \\
\hline 3. & Double fault condition to the operating table & $10^{-4}$ \\
\hline 4. & Degradation of AP insulation to $2 \mathrm{M} \Omega$ & $10^{-4}$ \\
\hline 5. & Double fault condition to the defibrillator & $5^{*} 10^{-5}$ \\
\hline 6. & Degradation of AP insulation to $3 \mathrm{M} \Omega$ & $5^{*} 10^{-5}$ \\
\hline 7. & Degradation of AP insulation to $5 \mathrm{M} \Omega$ & $3^{*} 10^{-5}$ \\
\hline
\end{tabular}

near $300 \mu \mathrm{A}$ and to a ventricular fibrillation probability of $94 \%$, is $380 \mathrm{k} \Omega$. In this case, the microshock probability is equal to $9.4^{*} 10^{-3}$. The value reported in the first row of Table 5 has to be intended as the maximum value of microshock probability that can be reached with the minimum value of insulation resistance which is not signalled by the insulation monitoring device. In fact, the applied part insulation failure is considered to occur 1 times every 100 procedures, whatever is the severity of insulation breakdown. This hypothesis is important to underline the importance of the dielectric strength tests to each insulation fulfilled by the fabricant of the MEE. In fact, the occurrences of undesired event reported in Table 2 have been evaluated considering the independence of failure behaviour of each insulation, if it were not so, the occurrence probabilities would be greater. Also non-destructive tests on insulation resistance have to be performed before the acceptance of the equipment in the operating theatre and periodically, as required by [9]. The insulation resistance tests should be performed in addition to the leakage current measurement. In fact it can be proved by model simulations that performing the APLC test on a BF-type applied part of the patient monitor with the insulation resistance to the mains part down to $51 \mathrm{k} \Omega$, the leakage current measured by the tester is $8 \mu \mathrm{A}$. So one cannot notice the deterioration occurred to the equipment under test. On the contrary this condition is severe and can lead to a not tolerable microshock probability during a surgical procedure, as shown in Table 5.

Another event considered is the double fault condition of the operating table. Though the microshock probability result is under the threshold of $10^{-3}$ an incorrect maintenance and inspection of the operating theatre could cause accidents. It is very important to maintain carefully the power supply cord to prevent its damage. For information on similar events see [17]. Moreover, if the table has an internal electrical power source it has to be disconnected from the mains source during a surgical procedure. These interventions prevent not only the microshock risk but also the burns due to the adoption of electrosurgery which are more likely to occur.

The IEC 60601-1 [8] refers that the probability of occurrence of undesired events listed in Table 2 would be less than those reported there. Improvements in 
design, more reliable components, better materials and the use of risk management processes would decrease the probability down to $2 \%$ for the occurrence of a single fault condition and $0.04 \%$ for the double fault condition and the degradation of the insulation of an applied part. These values would decrease the microshock probability under the tolerable limit accepted. So, failure rates of electrical insulations of medical equipment should be communicated to the responsible organizations, if it were not so, one should measure the insulation resistance before the acceptance of the medical equipment and also the interval between two inspections should be defined.

\section{Conclusions}

Operating theatres are locations where microshock risk has to be assessed to prevent the ventricular fibrillation due to dangerous leakage current that may flow through the patient. In this paper a method for the evaluation of the microshock probability is presented in order to investigate and to choose the mitigating interventions.

An electrical circuit model of a surgical layout composed by the electrical supply system, the patient, an operating table, a defibrillator and a patient monitor is shown. Undesired events have been identified by simulations of leakage currents flowing through the chest of the patient in different fault conditions.

The degradation of applied part insulations is the hazardous fault.

However, the microshock probability is tolerable if the occurrence of the event is equal to $0.04 \%$. The insulation resistance values should be known before the acceptance of a MEE and they should be periodically measured to prevent the increase of the microshock probability due to the ageing of the insulation. Though the MEEs have passed the electrical safety tests, a risk for patients to be victims of electroshock remains, so by measuring the insulation resistances of a MEE it is possible to know the microshock probability related to its use during a surgical procedure.

In conclusion, this method permits the probabilistic microshock risk assessment by simply measuring the resistance of the insulations of an applied part and then by simulating the surgical layout by its electrical circuit model.

\section{Acknowledgements}

The authors are grateful to the Clinical Engineering Unit of University Campus Bio-Medico of Rome for their support during measurements and to Eng. Simone Sepe for his valid support during the model development.

\section{References}

[1] G.L. Amicucci, L. Di Lollo, F. Fiamingo, V. Mazzocchi, G. Platania, D. Ranieri, R. Razzano, G. Camin, G. Sebastiani, P. Gentile, Electrical safety during transplantation. Transplantation Proceedings, Elsevier, 42, pp. 2175-2180, 2010. 
[2] G.L. Amicucci, F. Fiamingo, C. Mazzetti, Gli impianti elettrici ospedalieri: indicazioni costruttive e di utilizzo, in Italian, ISPESL Ed., Monograph, Supplement to Prevention Today, 1, 2008, pp. 19-54 -ISBN 9788889415444

[3] C.-Y. Yan, X.-J. Cai, Y.-F. Wang, H. Yu, Ventricular fibrillation caused by electrocoagulation in monopolar mode during laparoscopic subphrenic mass resection, Surgical Endoscopy, 25, 309-311, 2011.

[4] M.K. Chernovsky, J.E. Sipe, R.A. Ogle, Evaluation of health care operating rooms as wet/dry locations, The final report, The Fire Protection Research Foundation, 2010.

[5] D.H. Atkin, L.R. Orkin, Electrocution in the Operating Room, Anesthesiology, 38 (2), 181-183, 1973.

[6] G.L. Amicucci, F. Fiamingo, E. Zennaro, L. Poggi, Gestione del rischio per la protezione e la sicurezza dei sistemi elettrici a servizio delle strutture ospedaliere in Italian, VII edition of VGR 2012, Valutazione e Gestione del Rischio negli Insediamenti Civili ed Industriali, Pisa (Italy), October 3-5, 2012.

[7] E. Zennaro, C. Mazzetti, F. Fiamingo, G.L. Amicucci, Circuit model of medical equipment for electrical safety purposes, paper presented at the WIT $5^{\text {th }}$ International Conference on Safety and Security Engineering 2013, Rome (Italy), 2013.

[8] CEI EN 60601-1 "Medical electrical equipment - General requirements for basic safety and essential performance" $3^{\text {rd }}$ ed., 2007.

[9] IEC EN 62353: Medical electrical equipment - Recurrent test and test after repair of medical electrical equipment, $1^{\text {st }}$ ed., 2008.

[10] E. Zennaro, G.L. Amicucci, F. Fiamingo, C. Mazzetti, Circuit model of a medical equipment for electrical safety assessment, manuscript submitted for publication on International Journal of Safety and Security Engineering.

[11] IEC 60364-7-710: Electrical installations of buildings - Part 7-710: Requirements for special installations or locations - Medical locations, 2002.

[12] T. Kisielewicz, G.B. Lo Piparo, F. Fiamingo, C. Mazzetti, B. Kuca, Z. Flisowsky, Factors affecting selection, installation and coordination of surge protective devices for low voltage systems, Electric Power Systems Research, ISSN: 0378-7796, vol. 113, 2014.

[13] IEC/TS 60479-1: Effects of current on human beings and livestock - Part 1: General aspects, $4^{\text {th }}$ ed., 2005.

[14] A.B. Watson, J.S. Wright, J. Loughman, Electrical thresholds for ventricular fibrillation in man. Medical Journal of Australia, 1(24), 1179$1182,1973$.

[15] M. Laks, R. Arzbaecher, J. Bailey, A. Berson, S. Briller, D. Geselowitz, Will relaxing safe current limits for electromedical equipment increase hazards to patients? Circulation, 89 (2), 909-910, 1994.

[16] C.D. Swerdlow, W.H. Olson, M.E. O’Connor, D.M. Gallik, R.A. Malkin, M. Laks, Cardiovascular collapse caused by electrocardiographically silent 
106 Safety and Security Engineering VI

60-Hz intracardiac leakage current: Implications for electrical safety, Circulation, 99, 2559-2564, 1999.

[17] N.M. Courtney, E.P. McCoy, R.J. Scolaro, P.A. Watt, A serious and repeatable electrical hazard - compressed electrical cord and an operating table, Anaesthesia and Intensive Care, 34, 392-396, 2006. 